\title{
Presencialidad remota desde la perspectiva estudiantil y docente: un análisis de la División de Educación para el Trabajo de la Universidad Nacional, Costa Rica
}

Telepresence from the student and teacher perspective: an analysis of the Education for Work Division at Universidad Nacional, Costa Rica

A presencialidade remota na perspectiva de alunos e professores: uma análise da Divisão de Educação para o Trabalho da Universidad Nacional, Costa Rica

\begin{abstract}
Virginia Cerdas-Montano
Universidad Nacional

Heredia, Costa Rica

norma.cerdas.montano@una.cr

(D) ORCID: https://orcid.org/0000-0003-1705-4630

German González-Sandoval

Universidad Nacional

Heredia, Costa Rica

german.gonzalez.sandoval@una.cr

(1) ORCID: https://orcid.org/0000-0002-9149-7635

Silvia Elena Salas-Soto

Universidad Nacional

Heredia, Costa Rica

silvia.salas.soto@una.cr

(1) ORCID: https://orcid.org/0000-0002-1629-140X

Víctor Villalobos-Benavides

Universidad Nacional

Heredia, Costa Rica

victor.villalobos.benavides@una.cr

(D) ORCID: https://orcid.org/0000-0003-1290-3111

Recibido - Received - Recebido: 29 / 06 / 2021 Corregido - Revised - Revisado: 10 / 11 / 2021 Aceptado - Accepted - Aprovado: 25 / 11 / 2021

DOI: https://doi.org/10.22458/ie.v24i36.3612

URL: https://revistas.uned.ac.cr/index.php/innovaciones/article/view/3612

Resumen: La educación universitaria se enfrenta a una actualización metodológica ante los cambios suscitados por la crisis sanitaria generada por el virus responsable de la COVID-19, por tal razón desde la División de Educación para el Trabajo en la Universidad Nacional, se busca realizar un análisis del contexto y la mediación pedagógica suscitada a partir del 2020 y desde la perspectiva estudiantil y docente. Se analizan tres elementos medulares en el marco de un paradigma naturalista desde una metodología exploratoria, para identificar las ventajas y desventajas del trabajo en modalidad presencial remota, así como los posibles ajustes por realizarse en un eventual retorno presencial y la preferencia de modalidad de las personas participantes en este proceso de formación. Respecto a estos tres principales resultados se destacan ventajas señaladas tanto por el estudiantado como por el personal docente, las cuales se presentan a nivel personal, familiar, económico, de calidad de vida y salud, además de aspectos académicos, tal como el desarrollo de habilidades y capacidades para la vida profesional y para la continuidad de proyectos vocacionales, entre otros. Las desventajas se refieren a actividades domésticas, tareas múltiples, condiciones ergonómicas, interacción social y la conectividad. Además, dentro de los eventuales ajustes señalados están las condiciones laborales, la capacidad de adaptación al cambio, a la organización de horarios y de lo económico. El estudio concluye que el impacto del virus responsable de la Covid-19, a mediano y largo plazo, en la vida universitaria es inevitable y sus alcances afectarán la forma de organización institucional, la entrega de la docencia y los vínculos relacionales de sus miembros.
\end{abstract}

Palabras clave: Educación, Docencia, Formación Universitaria, Método de Enseñanza, Presencialidad Remota, Pandemia, Aprendizaje en línea. 


\begin{abstract}
Higher education is facing a methodological update due to the changes caused by the health crisis generated by the virus responsible for COVID-19; consequently, the Division of Education for Work at Universidad Nacional seeks to analyze the context and the pedagogical mediation arising since 2020 from the student and teacher perspective. Three core elements are analyzed within the framework of a naturalistic paradigm from an exploratory methodology to identify the advantages and disadvantages of working in a remote face-to-face modality, as well as the possible adjustments to be made in an eventual face-to-face return and the modality preference of the participants in this training process. Regarding these three main results, advantages are highlighted, indicated by both the students and the teaching staff, which are presented at a personal, family, economic, quality of life, and health level, in addition to academic aspects, such as the development of skills, capacities for professional life and for the continuity of vocational projects, among others. The disadvantages refer to household activities, multitasking, ergonomic conditions, social interaction, and connectivity. In addition, within the eventual adjustments, we have: working conditions, the ability to adapt to change, scheduled organization, and the economic situation. The study concludes that the impact of the virus responsible for Covid-19, in the medium-to-long term, in university life is inevitable, and its scope will affect the institutional organization, teaching practices, and bonding between the ones involved.
\end{abstract}

Keywords: Education, Teaching, University Training, Teaching Method, Telepresence, Pandemic, Online Learning.

Resumo: $O$ ensino universitário enfrenta uma atualização metodológica devido às mudanças provocadas pela crise sanitária gerada pelo vírus responsável pelo COVID-19, por isso a Divisão de Educação para o Trabalho da Universidade Nacional procura realizar uma análise contextual e pedagógica do tipo de mediação que surge a partir de 2020 na perspectiva do aluno e do professor. Três elementos centrais foram analisados sob um paradigma naturalístico e a partir de uma metodologia exploratória, para identificar as vantagens e desvantagens de se trabalhar na modalidade presencial remota, bem como os possíveis ajustes a serem feitos em uma eventual volta ao presencial e a preferência de modalidade das pessoas participantes neste processo formativo. Em relação a esses três resultados centrais, destacam-se vantagens, apontadas tanto pelo corpo discente quanto pelo corpo docente, que se apresentam ao nível pessoal, familiar, econômico, de qualidade de vida e de saúde, além de aspectos acadêmicos, como o desenvolvimento de aptidões e capacidades para a vida profissional e para a continuidade de projetos profissionais, entre outros. As desvantagens referem-se a atividades domésticas, multitasking, condições ergonômicas, interação social e conectividade. Além disso, entre os eventuais ajustes mencionados estão as condições de trabalho, a capacidade de adaptação às mudanças, a organização dos horários e a condição econômica. O estudo conclui que o impacto do vírus responsável pela Covid-19, em médio e longo prazo, na vida universitária é inevitável e sua abrangência afetará a organização institucional, a oferta de ensino e os laços relacionais de seus membros.

Palavras-chave: Educação, ensino, treinamento universitário, método de ensino, presencialidade remota, pandemia, aprendizado online.

\title{
INTRODUCCIÓN
}

La educación universitaria en los contextos actuales ha tenido que hacer ajustes significativos para ofertar las diferentes carreras, (Ocaña, Valenzuela y Morillo, 2020). Dichos ajustes se han venido desarrollando de manera precipitada en la última década, principalmente con la incorporación de las tecnologías a los entornos de aprendizaje. (De Pablos, Bravo, López y Lázaro, 2019). Aunado a esto, también se marcan los cambios sociales, económicos, ambientales de los cuales la generación actual es testigo. En este escenario dinámico y cambiante se presenta otro desafío a nivel mundial: la pandemia del virus responsable de la COVID-19.

El contexto educativo costarricense, al igual que del resto del mundo, se vio afectado por la pandemia, cuyos primeros contagios en el país se dieron en marzo del 2020. A partir de este momento, los ajustes que, si bien se percibían como vertiginosos, se aceleran de manera significativa. Como resultado de este proceso de cambio inesperado, surge el desafío de abrir espacios aptos para el aprendizaje según la nueva forma de mediación pedagógica en el contexto universitario. Tanto el estudiantado como el equipo docente enfrentan un cambio radical en la forma tradicional de ofertar las carreras en la mayoría de las facultades de la Universidad Nacional, cuyo estudiantado, aunque ya hubiese incorporado componentes tecnológicos, siempre recurría a la presencialidad como parte distintiva de sus procesos de aprendizaje. 
En este sentido la División de Educación para el Trabajo (DET) del Centro de Investigación y Docencia en Educación (CIDE) de la Universidad Nacional (UNA), repentinamente se ve de cara ante el desafío de ofertar sus carreras de grado y posgrado en la modalidad presencial remota adaptada por la UNA, con el fin de proteger la vida de la comunidad educativa ante la amenaza de la pandemia. En este marco de acción el Consejo Académico (CONSACA), como órgano superior responsable de promover, coordinar, articular, integrar y orientar el quehacer académico de la Universidad, emite un acuerdo el 20 de marzo de 2020 (UNA-CONSACA-ACUE-045-2020, comunicado institucional) en donde se formaliza la presencialidad remota como la modalidad para ejercer la docencia. Dicha modalidad se define como "la posibilidad de participar de procesos educativos desde lugares geográficamente distribuidos y de manera sincrónica o asincrónica" (Considerando 2).

En este contexto, la DET se pregunta cuál es la opinión estudiantil y de las personas docentes acerca de las ventajas y desventajas de la experiencia de la presencialidad remota. Por lo tanto, esta investigación plantea como objetivo general analizar, desde la percepción estudiantil y docente, las ventajas y desventajas de la presencialidad remota y los ajustes por realizar un eventual retorno a la presencialidad. Como objetivo específico se identifican, desde la percepción estudiantil, las ventajas y desventajas de la presencialidad remota como sujetos receptores de la oferta educativa, así como los ajustes a implementar ante un eventual retorno a la presencialidad. Otro objetivo específico planteado, consiste en determinar la percepción del personal docente sobre las ventajas y desventajas de la presencialidad remota y las posibles implicaciones de un eventual retorno a la presencialidad. Adicionalmente se consulta a la población participante sobre su preferencia de modalidad de trabajo para el II ciclo lectivo 2021.

A partir de los cambios acelerados y de la incertidumbre generada por la situación sanitaria, en la población estudiantil y en el cuerpo docente, se hace indispensable valorar las acciones implementadas como respuesta inmediata a la necesidad de darle continuidad al proceso de aprendizaje, garantizando los principios fundamentales de la formación universitaria, ya que este proceso de evaluación del contexto forma parte de la misión histórica de la UNA, comprometiendo a la institución con "la investigación sistemática de la realidad costarricense, dentro de un marco de solidaridad y armonía entre el ser humano y la naturaleza; también le compromete a fortalecer una cultura humanista y a contribuir en la creación de una sociedad más solidaria, próspera, justa y libre." (Estatuto Orgánico, 2015. p. 1).

Los retos y desafíos enfrentados por las universidades de América Latina ante la situación de emergencia y las estrategias para enfrentar el cambio ha sido motivo de discusión institucional: "las medidas para promover el distanciamiento social crearon un proceso de digitalización forzada, lo cual coaccionó los mecanismos de pedagogía a los de la teleeducación con el objetivo de garantizar la sostenibilidad de las clases y las labores académicas y administrativas de las instituciones de educación superior" (Rodríguez, 2021, Párr. 2).

Las instituciones de educación superior han venido enfrentando dificultades debido al cambio en las modalidades de entrega de la docencia, por ejemplo, Kikut (2020) señala "Si bien esta institución venía trabajando desde hace varios años en la virtualización de algunos cursos, la gestión de migrar toda la oferta académica a esta modalidad no resultó una tarea fácil ni para el profesorado ni para el estudiantado" (p. 3). Esta ha sido la experiencia de la Universidad de Costa Rica. En general, de acuerdo con la mayoría de las instituciones superiores "lo que ha venido sucediendo no es un proceso de innovación cuidadosamente diseñado y validado antes de generalizarse, sino una masiva respuesta que va construyéndose en la práctica de acuerdo con las posibilidades de cada institución" (Acosta, Ortega, y Díaz. 2020. p. 3).

Para el desarrollo de los objetivos mencionados anteriormente, esta investigación busca analizar los resultados desde diferentes acercamientos conceptuales. Interesa en esta investigación abordar la importancia de comprender la presencialidad remota. Tijo (2020) se refiere a la enseñanza remota de emergencia como el espacio virtual simulador de las clases presenciales en sus horarios respectivos. Según el autor los profesores se reúnen con estudiantes para desarrollar el trabajo en clase, modalidad 
adaptada a la nueva realidad, generada por la emergencia sanitaria. En este sentido, la enseñanza remota no cuenta con el debido tiempo de planificación, propios de los cursos de modalidad en línea.

Por otro lado, Ibáñez (2020) caracteriza a la educación remota de emergencia como una educación obligada a adaptar sus métodos a una nueva modalidad, en un lapso muy corto para continuar impartiendo clases a los estudiantes. Como objetivo principal se plantea la necesidad de trasladar los contenidos impartidos de forma presencial a un espacio virtual.

Es importante señalar que la presencialidad remota constituye una adaptación de planes de estudio diseñados para ser impartidos de manera presencial, los cuales se desarrollaron de manera virtual debido a la emergencia sanitaria. Así los planes de estudio de la modalidad virtual quedaron ajenos a los extensos procesos de diseño instruccional, propios de nuestras instituciones. De esta manera Shimshon y Dye (2020) opinan sobre lo que ellos Ilaman "aprendizaje remoto", definiéndolo como una estrategia para realizar cambios que normalmente llevan años en tan solo semanas, como respuesta a la COVID-19. Según los autores, esta modalidad busca replicar la enseñanza, ya establecida para la presencialidad, en espacios virtuales.

Desde la presencialidad remota, que emerge de manera vertiginosa, se evidencia una serie de situaciones características de este nuevo entorno educativo. Un factor se refiere a la socialización a través de entornos virtuales, desafiando a las personas a tener una interacción social, manteniendo los procesos de comunicación activos; es decir, mantener los canales abiertos para externar emociones y necesidades propias de los procesos de aprendizaje. En este sentido, Rodríguez (2018) y Ortiz y Neira (2018) proponen el diseño de los cursos para modalidades virtuales y a distancia como modelos tendientes a favorecer la interacción social de las personas participantes.

Por otro lado, el concepto de exclusión, según la Comisión Económica para América Latina y el Caribe (CEPAL, 2020), hace referencia a la desigualdad exacerbada por la pandemia en los contextos actuales, en donde se carece de las condiciones adecuadas para las modalidades virtuales y se dificulta la continuidad o permanencia de las personas estudiantes en los programas de estudio. En este sentido, la UNA, a través del redireccionamiento de los presupuestos del Fondo de Fortalecimiento y Renovación Académica (FFRA), la Vicerrectoría de Vida Estudiantil en conjunto con la Vicerrectoría de Docencia, la Proveeduría Institucional, la Dirección de Tecnologías de Información y Comunicación y el Programa de Gestión Financiera, desarrolla una estrategia de apoyo al estudiantado con el fin de facilitar 3.500 planes de conectividad a internet y el préstamo de 500 tabletas electrónicas, para facilitar a los estudiantes en condiciones especiales la continuidad del proceso educativo. Dicho programa se conoce con el nombre de Proyecto Conectando UNA Población Estudiantil (UNA-CONSACA-ACUE-118-2020, comunicado institucional).

Por otro lado, se torna relevante la valoración del contexto familiar, la cual, según Duarte (2019), representa un factor clave para el estudiantado, pues constituye su red de apoyo inmediata. Esta a su vez repercute en la sociedad, por tanto, el Estado debería de garantizar condiciones idóneas para las familias, principalmente para las de mayor vulnerabilidad y expuestas a conflictos emocionales y psicológicos, asociadas con la pobreza extrema.

Otro aspecto importante para las personas estudiantes se refiere a las actividades domésticas, las cuales son producto de la dinámica propia de cada entorno familiar y con su propia forma de organizar las tareas del hogar. Según Pablo (2020), la pandemia implicó reajustar la carga académica para el desarrollo de los cursos; por lo tanto, la calidad, cantidad y distribución de horas se vieron alteradas, transformando la dinámica familiar.

Según Pablo (2020) la condición de género también tiene sus implicaciones: "el entrecruzamiento de datos relacionados con el ámbito doméstico, y teniendo en cuenta el alto componente femenino del sector, permite identificar una serie de variables que demuestran las dificultades en las que se ve envuelta la 
tarea docente en medio de la cuarentena" (p. 926). La carga cultural se asocia con la condición de género y representa una dificultad en doble vía, que afecta tanto a la población estudiantil como al profesorado.

Es importante recalcar que emergen conceptos compartidos, tanto por el contexto estudiantil como por el docente, tales como el de "la nueva realidad", asociado con el resguardo de la vida y la salud de la comunidad educativa. En este sentido según la CEPAL (2020), el ámbito educativo de los países de la región adoptó modalidades de aprendizaje a distancia y virtuales, y se dio la movilización de educadores para apoyar a las comunidades brindando una atención integral al estudiantado. Ante estas medidas sanitarias, la UNA promueve el teletrabajo para sus funcionarios, así como la modalidad de presencial remota para ofertar las carreras.

Entre los aspectos relevantes para este estudio se destaca la calidad de vida, definida por Aguilar (2018), como un proceso multifactorial, en donde intervienen elementos biológicos, sociales, ambientales y emocionales entre otros. Enfrentar el cambio genera estrés y requiere un enfoque distinto al de la psicología clínica clásica. Por tanto, para tener calidad de vida es necesario identificar los procesos desencadenantes de alteraciones negativas en el organismo y contrarrestarlos mediante acciones integrales tendientes a mejorar la salud.

Por otro lado, en la adquisición de conocimiento intervienen factores emocionales. Según Smith (2019), el aprendizaje cognitivo requiere de procesos emocionales y sociales, por tanto, el cómo la persona estudiante y el equipo de profesores se sientan durante el proceso afectará la manera de recuperar y procesar la información recibida. Por otro lado, los procesos sociales constituyen una parte esencial del proceso cognitivo y pueden potenciar o reducir la capacidad de aprendizaje.

Ante estos cambios, situaciones emocionales y la procura de la calidad de vida se requiere desarrollar la capacidad de adaptación al cambio. Pablo (2020) se refiere a una adaptación vertiginosa, provocada por la pandemia, a nuevas formas de trabajo, las cuales derivan nuevos saberes, emocionalidades y prácticas de virtualización, asumidas de manera resignada tanto por el colectivo de docentes como por la población estudiantil.

Por otro lado, se evidencia la importancia del desarrollo de las habilidades tecnológicas, conceptualizadas, según Inciarte (2008), como "la expresión didáctica de la profesión, a partir del concepto de las habilidades generalizadas, los núcleos de conocimientos y los valores profesionales que le permiten al docente actuar de manera creativa, pertinente, efectiva y eficaz en el diseño de contenidos" (p. 34). lo anterior al trasladarse al rol del estudiantado, permitiría la incorporación efectiva al proceso de aprendizaje. En todo caso las habilidades tecnológicas son necesarias tanto para el equipo docente como para el estudiantado.

Aunado a lo anterior, se suma la necesidad del acceso de la comunidad universitaria a una adecuada conectividad, para enfrentar las estrategias vinculadas a la modalidad emergente. Algunas entidades, tal como la Organización de las Naciones Unidas para la Educación, la Ciencia y la Cultura. (UNESCO, 2019), se plantean asuntos vinculados con la cantidad y calidad del equipamiento, el nivel y el alcance de la conectividad y la disponibilidad de soporte técnico para la actualización, mantenimiento y renovación de los equipos de los centros educativos; sin embargo, es posible establecer estos mismos aspectos como parámetros para la conectividad en el hogar.

Al respecto, el Consejo Nacional de Rectores (CONARE) declara el 2021, como el año de la conectividad como derecho humano universal de las Universidades Públicas, para el fortalecimiento de una sociedad más justa y el desarrollo humano sustentable. En esta misma línea, la investigación de Castellano (2020), realizada con la población de estudiantes del CIDE de la UNA, concluye que "dispone de un alto nivel de capital tecnológico objetivado, que se manifiesta en un elevadísimo índice de apropiación individual de los recursos tecnológicos y en un destacado rango de acceso de conexión a Internet" (p. 341). 
Relacionado con están las condiciones ergonómicas o de acceso. Acerca de esto, la Comisión Económica para América Latina y el Caribe (CEPAL, 2020) se pronuncia en relación con las formas de acceso disponibles, su diversificación y complejidad, destacando la importancia del acceso, pero sobre todo de la calidad y el aprovechamiento que se le pueda dar.

Por otra parte, en el contexto propiamente docente las condiciones laborales constituyen un aspecto relevante. Al respecto el investigador Pablo (2020) menciona que "la jornada laboral docente se ha completado desde el domicilio y fue modificada tanto en su calidad como en la cantidad y distribución de horas. Debido a esta situación, las tareas domésticas y la dinámica familiar también se han transformado" (p. 924).

Finalmente, según León y Zúñiga (2019) la mediación pedagógica, concebida como un proceso durante el cual se potencia la creatividad, a través de espacios de aprendizaje agradables y facilitadores de una comunicación en doble vía y, por lo tanto, un espacio en el cual, a pesar de su trabajo desde la virtualidad, la persona docente propicia una interacción efectiva con la persona estudiante para la asimilación de los nuevos saberes.

Los aspectos analizados en este estudio se enmarcan en condiciones particulares y son generados principalmente por la pandemia y repercuten directamente en la forma de ofertar las carreras de la DET; por tanto, en el análisis de los resultados se profundiza en las ventajas y desventajas de la presencialidad remota, así como en los ajustes a implementar al momento de un posible retorno de la comunidad universitaria.

\section{MATERIALES Y MÉTODOS}

El paradigma seleccionado para la investigación es naturalista, se parte de las fortalezas representadas por esta perspectiva y de las diversas formas de acercarse a una misma realidad para la comprensión de un fenómeno, "se centra en el estudio de los significados de las acciones humanas y de la vida social." (Barrantes, 2014 p. 82). De ahí que este estudio se enfoca en comprender la realidad de las personas y su contexto. El enfoque es cualitativo, y cumple con los objetivos propuestos, desde una perspectiva inductiva para plantear el problema; también se explora y se establece un análisis teórico en torno a la realidad social investigada. El método empleado para responder al tema, al problema y a los objetivos planteados en esta investigación corresponde al exploratorio, lo que presupone aproximaciones a fenómenos novedosos, tal como es la pandemia del Covid-19; fenómeno acerca del cual no se pretende llegar a conclusiones generalizadas, pero sí contribuir para una mayor comprensión del tema. (Hernández, Fernández, y Baptista, 2014).

La selección de los participantes se desarrolla con un criterio de conveniencia, acorde con la naturaleza de este tipo de estudios cualitativos. En este sentido se consulta a 388 estudiantes y 27 docentes de la División de Educación para el Trabajo, quienes respondieron un cuestionario en línea de manera abierta y voluntaria. Posteriormente se realizan grupos focales según una selección por conveniencia para ambas poblaciones. Entre los criterios para conformar estos grupos se establecen aspectos como la flexibilidad de horarios y las posibilidades de asistir a sesiones sincrónicas. De las 388 personas estudiantes se reciben 108 respuestas y 14 de personas docentes.

La naturaleza de este estudio prioriza el análisis del contexto y de sus condiciones, lo cual permite una compresión global del fenómeno social. Finalmente, se realizan dos grupos focales con ambas poblaciones participantes, a partir de los cuales se busca profundizar en las ventajas y limitaciones de la presencialidad remota y los posibles ajustes necesarios ante un eventual retorno a la presencialidad inmediata, así como realizar un análisis comparativo entre las respuestas del estudiantado y las del personal académico 
acerca de la modalidad de su preferencia. Al respecto, según los autores citados, cuando se examina un tema poco estudiado, no abordado antes, el cual despierta muchas dudas e ideas vagamente relacionadas, se requiere de un estudio exploratorio (Hernández, Fernández y Baptista, 2014).

Como técnica de recolección de información se utiliza un cuestionario en línea, el cual se compone de tres preguntas de respuesta abierta y una cuarta pregunta de respuesta semicerrada, utilizando el mismo formato para ambas poblaciones consultadas. De acuerdo con Hernández, Fernández y Baptista (2014), esta técnica es la más utilizada para recolectar datos de fenómenos sociales. Ambos grupos focales se encuestaron mediante un instrumento donde se plantean cinco preguntas acerca de la presencialidad remota, sus ventajas, desventaja y ajustes.

Para el análisis de la información recolectada, se codifican los datos cualitativos, a partir de lo expresado por los encuestados. A los datos se les asigna categorizaciones y subcategorizaciones y se registran patrones de incidencia para la reducción de la información, para luego triangularla con la teoría para sacar conclusiones. Además, se efectúa un análisis comparativo entre las respuestas del estudiantado y las del personal académico acerca de preferencia por modalidad.

Por último, considerando el enfoque de investigación y para cumplir los criterios de rigurosidad y validez expresados por Bisquerra (2014), se establecen parámetros tales como la credibilidad, la cual se genera mediante la triangulación de los datos del cuestionario, el grupo focal y los supuestos teóricos, a partir de los cuales se analiza el fenómeno en estudio.

\section{DISCUSIÓN DE RESULTADOS}

La presencialidad remota ha representado para la población estudiantil y docente la realización de ajustes en los procesos de aprendizaje, pero también en la cotidianidad de la vida personal y familiar. El impacto de los cambios en todos los ámbitos del desarrollo humano es apenas incipiente, sin embargo, existe evidencia de factores considerados como representativos de este cambio, tales como: interacción social, contexto familiar, actividades domésticas, calidad de vida, condición de género, entre otros. Los procesos de aprendizaje en el contexto universitario, tal y como lo indican De Pablos, Bravo, LópezGracia, y Lázaro (2019), están sometidos a ajustes para mantener la vigencia de la oferta educativa. La pertinencia de dicha oferta se considerada una condición necesaria para responder a la demanda del contexto social donde se desarrolla. Con la aparición de la pandemia, la Universidad Nacional se vio obligada a replantear la forma de entrega de la docencia, para resguardar la seguridad de la población estudiantil y la del profesorado. En este sentido, la consulta sobre ventajas y desventajas de la presencialidad remota como modalidad de mediación pedagógica en la DET y los ajustes a un posible retorno a la presencialidad arrojan datos que denotan la percepción de las personas participantes desde dos dimensiones, la académica y la personal. La primera giró en torno a los procesos de formación universitaria y la segunda en torno al rol desempeñado por cada persona dentro de su entorno familiar. Estas dimensiones surgen a partir de la consulta y se asumió como parte de los hallazgos de la investigación.

Acerca del contexto familiar, el estudiantado considera una ventaja la posibilidad de permanecer en el hogar, pues se favoreció el contacto interpersonal con los familiares y facilitó una mayor participación en las actividades de otros miembros del núcleo familiar. En la modalidad presencial la persona estudiante no gozaba de la posibilidad de involucrarse tanto en las dinámicas del hogar. Al respecto, destacan las expresiones de algunas personas estudiantes consignadas en la tabla 1. 
TABLA 1

Contexto Familiar

\begin{tabular}{|c|c|}
\hline Informante & Frase \\
\hline Estudiante 15: & $\begin{array}{l}\text { "Estar más cerca de mi familia, así como poder hacer otras actividades que no tenía la disponibilidad } \\
\text { de hacer mientras estaba fuera de mi hogar" }\end{array}$ \\
\hline Estudiante 2: & $\begin{array}{c}\text { "Más tiempo de convivencia familiar, cumplimiento de tareas hogareñas, más tiempo para realizar } \\
\text { los trabajos y organizarme, tiempo para dedicar a un área física" }\end{array}$ \\
\hline
\end{tabular}

Fuente: Consulta a estudiantes y docentes. Elaboración propia (2021).

Lo anterior se manifestó como una ventaja en tanto el contexto familiar representó una de las redes de apoyo emocional más relevante para la persona estudiante, coincidiendo con lo expuesto por Duarte (2019). En este sentido, las actividades domésticas representan un vínculo de convivencia familiar, propio de cada hogar (Pablo, 2020). La pandemia hizo necesario realizar algunos reajustes, tanto en los horarios como en los espacios físicos donde realizar las labores académicas, las cuales en la presencialidad se llevaban a cabo dentro de la institución académica.

Otro aspecto considerado como una ventaja por la población estudiantil se refiere a la calidad de vida, vinculada a la posibilidad de realizar otras actividades como parte de su desarrollo integral, por ejemplo: el deporte, la recreación, la espiritualidad, entre otras. Estas actividades favorecieron la reducción del estrés por agotamiento y el desgaste mental causado por la pandemia. De acuerdo con lo manifestado por el estudiantado, su salud en general ha mejorado, proporcionándole mayor bienestar y calma. Algunas expresiones de los estudiantes se presentan en la tabla 2.

TABLA 2

Calidad de Vida

\begin{tabular}{lc}
\multicolumn{1}{c}{ Informante } & Frase \\
Estudiante 51: & "Realizar actividades recreativas" \\
Estudiante 27: & "Realizar actividades para mejorar mi salud" \\
\hline & Fuente: Consulta a estudiantes y docentes. Elaboración propia (2021).
\end{tabular}

Así las cosas, se percibe una actitud positiva en el estudiantado frente a la presencialidad remota, en el tanto les brindó espacios para mejorar su calidad de vida, lo cual, según Aguilar (2018), constituye un aspecto clave para enfrentar procesos de cambio.

En lo referente al desplazamiento, el estudiantado asegura gozar de un considerable ahorro de tiempo en el traslado desde su residencia hasta la universidad y viceversa; además de ello, disfruta de más seguridad personal, ya que evita los riesgos asociados al regreso a casa durante la noche. Esto es especialmente cierto para quienes residen en sitios donde los índices de delincuencia son muy altos (ver tabla 3). Por otra parte, la presencialidad remota evitó a los estudiantes de sectores muy alejados, tales como la zona peninsular, norte y sur, la necesidad de desarraigo de sus familias y comunidades. A modo de ejemplo se consignan las siguientes frases etnográficas:

TABLA 3

Desplazamiento

$\begin{array}{lrl}\text { Informante } & \text { Frase } \\ \text { Estudiante 71: } & \text { "Debería viajar a la sede y volver en la misma semana desde Talamanca, no cuento con beca, dependo } \\ \text { de los ingresos de mi esposo y sería muy difícil la inversión." }\end{array}$


La consideración del desplazamiento, en relación con el tiempo de permanencia en las instalaciones de la universidad y los traslados dentro del campus, implica una ventaja para el estudiantado, pues no debe esperar mucho tiempo entre una jornada y otra, y además realiza un menor gasto económico, paga menos pasajes, no debe costear estadías prolongadas, se reduce el costo de servicios, alimentación, materiales, libros, antologías o fotocopias de lecturas. Esta adaptación positiva al proceso de cambio concuerda con lo expuesto por Pablo (2020). Al ahorrar se le abre al estudiante la posibilidad de colaborar económicamente con hogar. Algunas de sus opiniones al respecto se registran en la tabla 4.

TABLA 4

Factores económicos

\begin{tabular}{|c|c|}
\hline Informante & Frase \\
\hline Estudiante 13: & $\begin{array}{c}\text { "Las ventajas han sido más de tipo económicas, ya que por el hecho de vivir en Heredia significaba } \\
\text { tener que pagar alquiler, comida, agua y luz, entre otros gastos" }\end{array}$ \\
\hline Estudiante 42: & "No gasto en transporte y puedo invertirlo en ayudar a mi familia" \\
\hline
\end{tabular}

Fuente: Consulta a estudiantes y docentes. Elaboración propia (2021).

Se consideró, como una de las ventajas académicas, la mediación pedagógica en la modalidad de la presencialidad remota, ya que el estudiantado percibió el desarrollo de las sesiones de la clase de una forma más concreta, tanto en relación con la extensión del tiempo, como como con los contenidos. Además, se percepción una mayor flexibilidad en la mediación al considerarse las condiciones individuales del estudiantado, sacando mayor provecho de las horas de atención individual, lo cual conduce a un seguimiento docente más individualizado, tal como se mencionó en el referente teórico. La virtualidad de la docencia permitió interacciones efectivas, promoviendo la asimilación de nuevos saberes, tal y como lo menciona León y Zúñiga (2019). De estos elementos positivos se pueden observar las siguientes frases de la tabla 5.

TABLA 5

Mediación Pedagógica

$\begin{array}{ll}\text { Informante } & \text { Frase } \\ \text { Estudiante 8: } & \text { "La flexibilidad de los profesores y profesoras a considerar las condiciones individuales de cada estu- } \\ \text { Estudiante 63: } & \text { diante. También ha sido un poco más sencilla la organización del tiempo" }\end{array}$

Fuente: Consulta a estudiantes y docentes. Elaboración propia (2021).

La consulta a estudiantes reflejó un factor preferente de acceso a la conectividad desde sus hogares, ya que señalaron que en las instalaciones del CIDE las redes tecnológicas internas tienen la característica de ser inestables. En este sentido es relevante señalar una severa situación que atenta con lo promulgado por el CONARE, al determinar este el año de la conectividad como derecho humano universal de las Universidades Públicas con la finalidad de fortalecer una sociedad más justa y el desarrollo humano sustentable, quedando en evidencia que a lo interno de la UNA no se garantiza dicha conectividad y que un sector de la población estudiantil goza de mejores condiciones en sus lugares de residencia. Por otra parte, señalaron que existe mayor accesibilidad a los contenidos y recursos didácticos virtuales que han quedado registrados en las diferentes plataformas en línea y ofrecen posibilidad de repaso pronto e inmediato indicando que tienen mayor facilidad de visualizar las clases de repaso, así como tener más accesibilidad en clases a información extra en la web.

Adicionalmente, la posibilidad de asumir actividades laborales y los proyectos vocacionales toman mayor relevancia, por lo que se manifestó la posibilidad de acceder a la continuidad de los estudios, en algunos casos porque pausaron su formación universitaria o bien únicamente cursaban el plan de manera parcial. En ese mismo sentido se expresó también de manera positiva la flexibilidad horaria, lo que 
a su vez se relaciona con la posibilidad de llevar a cabo actividades laborales para sostener sus propios estudios, así como apoyar a sus familias, coincidiendo con lo expuesto por Pablo (2020). Se comparten algunas de las frases representativas en la tabla 6.

TABLA 6

Flexibilidad Horaria

$\begin{array}{lrl}\text { Informante } & \text { Frase } \\ \text { Estudiante 33: } & \text { "Vivo lejos y desde casa se me hace más fácil poder recibir clases, estoy segura con mi familia, no hay } \\ \text { exposición, y no tengo que pagar alquiler para poder asistir a la universidad" }\end{array}$

Se identificó el desarrollo de habilidades de tipo profesional (tabla 7) debido a que el estudiantado hizo énfasis en la adquisición de destrezas para el uso de herramientas tecnológicas, lo que permitió crear conciencia sobre el derecho universal a la conectividad, además el conocimiento de herramientas tecnológicas potencia el desarrollo de habilidades de aprendizaje (Inciarte, 2008).

TABLA 7

Desarrollo de Habilidades

\begin{tabular}{|c|c|}
\hline Informante & Frase \\
\hline Estudiante 94: & "Conocer más sobre herramientas tecnológicas" \\
\hline Estudiante 53: & $\begin{array}{c}\text { "A pesar de las dificultades de conectividad ha sido una gran experiencia, ya que me ha tocado explo- } \\
\text { rar y desarrollarme más en el área tecnológica que antes no manejaba y cada día he logrado adquirir } \\
\text { más conocimientos." }\end{array}$ \\
\hline
\end{tabular}

En este sentido, destaca el autoaprendizaje y el compromiso con los procesos personales, habilidades de comunicación, desarrollo de la creatividad y, en el área intrapersonal, el autoconocimiento, la autorregulación y la autogestión. En algunos casos se reportó la mejora en el rendimiento académico, la "autonomía", "mayor organización del tiempo, más comodidad" y la "autoregulación del aprendizaje" (Inciarte, 2008 y Smith, 2019).

Finalmente, para el estudiantado es importante el tema de la seguridad personal, debido a que tiene la posibilidad de evitar el riesgo de contagio durante la pandemia CEPAL (2020), lo cual se ilustra en la tabla 8.

TABLA 8

Seguridad Personal

\begin{tabular}{l} 
Informante $\quad$ Frase \\
Estudiante 18: "poder mantener mi familia segura ya que son de alto riesgo" \\
\hline Fuente: Consulta a estudiantes y docentes. Elaboración propia (2021).
\end{tabular}

Por otra parte, el colectivo estudiantil identifica las desventajas vivenciadas en la presencialidad remota, entre ellas las tareas del hogar o la misma dinámica familiar. Según el contexto de cada persona estudiante, algunas situaciones pueden representar desventajas, tal como el ruido del ambiente, el cual puede entorpecer el desarrollo de su proceso de aprendizaje, causando distracción. Asimismo, se pronunció acerca de la simultaneidad de las tareas que se pueden realizar mientras se reciben las clases, por lo que en algunos casos la calidad del proceso de aprendizaje se ve afectado negativamente. Así, según Pablo (2020) la pandemia transformó la docencia y de igual manera la dinámica familiar (tabla 9). 
TABLA 9

Tareas del Hogar

\begin{tabular}{|c|c|}
\hline Informante & Frase \\
\hline Estudiante 37: & "más tiempo para dejar en orden las cosas de la casa y de la comida de mi familia" \\
\hline Estudiante 65: & "esto me ha traído dificultades ya que demanda mucho tiempo el estudio y la maternidad en conjunto \\
\hline
\end{tabular}

La condición física también representó una desventaja, debido al cansancio experimentado luego de actividades muy prolongadas, por lo cual también se requiere valorar espacio físico personal donde se lleva a cabo el proceso de aprendizaje. Asegurar adecuadas condiciones del espacio físico mediante la iluminación, la ventilación, la privacidad, es relevante para realizar actividades académicas, que facilita la capacidad de concentración (CEPAL, 2020).

Otra desventaja identificada fue la limitada posibilidad de interacción social, dificultando el desarrollo de habilidades de comunicación. Por tratarse de un proceso virtual no se posibilita la proximidad física y la percepción sensorial de la otra persona (tabla 10).

TABLA 10

Interacción Social

\begin{tabular}{lc} 
Informante & Frase \\
Estudiante 18: & "Desventaja social" \\
\hline Fuente: Consulta a estudiantes y docentes. Elaboración propia (2021).
\end{tabular}

Otra desventaja, reconocida como producto de la presencialidad remota, se encuentra en la pérdida de contacto social, minando las habilidades de comunicación y gestión de emociones. De acuerdo con Rodríguez (2018) y Ortiz y Neira (2018), el diseño de los cursos para desarrollarse bajo modalidad virtual y a distancia debe de favorecer la interacción social de sus participantes; los docentes podrían plantear actividades virtuales donde se dé oportunidad para el diálogo, la participación y otras formas de interacción para dar origen a comunidades del conocimiento. En estos espacios programar de manera intencionada y con los roles claramente establecidos para cada uno de los agentes del proceso formativo.

El limitado acceso a la conectividad o su total carencia en algunos casos, así como la calidad de los servicios públicos de conexión en sus lugares de residencia han perjudicado los procesos de aprendizaje (tabla 11). Ante lo cual la UNESCO (2019) plantea el alcance de la conectividad como un tema de reflexión para los centros educativos.

Tabla 11

Conectividad

\begin{tabular}{|c|c|}
\hline Informante & Frase \\
\hline Estudiante 55: & $\begin{array}{c}\text { "La educación no es lo mismo, ya sea por fallas de internet, ruido en la casa, son factores que influyen } \\
\text { a la hora de recibir la clase y no se puede recibir toda la información necesaria" }\end{array}$ \\
\hline Estudiante 27: & $\begin{array}{l}\text { "La verdad, aunque uno ha llegado a aprender la teoría, no encuentro alguna ventaja, pues nos quita- } \\
\text { ron mucho ejemplo visual y nos faltó la práctica" }\end{array}$ \\
\hline
\end{tabular}

Fuente: Consulta a estudiantes y docentes. Elaboración propia (2021).

Ante el eventual regreso a las aulas, el estudiantado necesitará realizar ciertos ajustes. Un ejemplo de esto se refiere a la población que labora, la cual se verá obligada a realizar cambios de horarios o renunciar. Esto implica el abandono de una u otra tarea por parte de algunas personas estudiantes vinculadas o aspirando a incorporarse al mercado laboral Pablo (2020). Lo cual se refleja en la tabla 12. 
Tabla 12

Condición Laboral

\begin{tabular}{l} 
Informante \\
Estudiante 57: \\
Estudiante 88: \\
\hline Fuente: Consulta a estudiantes y docentes. Elaboración propia (2021).
\end{tabular}

Existe en el estudiantado una preocupación relativa a la capacidad de adaptación al cambio (tabla 13), que implica el abandono de la comodidad del hogar al llevar a cabo su aprendizaje, para regresar a las aulas, a lo cual se suma la dificultad para organizar horarios y todo lo implicado en el traslado (tiempo, dinero, clima, otros) (Pablo, 2020).

Tabla 13

Tiempo

Informante

Estudiante 3:

Estudiante 54:

\section{Frase}

"Económico, por que viajaría un poco más y antes no lo hacía y de tiempo."

"Menos tiempo de movilización"

Fuente: Consulta a estudiantes y docentes. Elaboración propia (2021).

En la consulta se evidenciaron elementos relacionados con la condición de género (tabla 14), pues las madres deben asegurar el cuido de sus hijos e hijas o de las personas adultas mayores a su cargo antes de acudir a las aulas. La condición de género coloca en desventaja principalmente a las mujeres estudiantes Pablo (2020).

TABLA 14

Condición de Género

\begin{tabular}{ll} 
Informante & Frase \\
Estudiante 19: & $\begin{array}{l}\text { "El cuido de mi hijo menor, se requiere muchísimo más tiempo para desplazarse, y más tiempo para } \\
\text { dejar en orden las cosas de la casa y de la comida de mi familia" }\end{array}$ \\
Estudiante 27: & "Sería el cuido de mi hija y buscar para donde irme por motivos de que vivo lejos de la universidad" \\
\hline & Fuente: Consulta a estudiantes y docentes. Elaboración propia (2021).
\end{tabular}

Los principales cambios se refieren a aquellos relacionados con las medidas sanitarias para proteger la salud en la presencialidad del aula, así como los referidos a emociones experimentadas ante la necesidad de adaptarse nuevamente al aula, o ante el desarraigo, en el caso de estudiantes que deben abandonar su hogar y comunidad para trasladar su domicilio al lugar donde se ubica la unidad académica a la cual se incorpora (tabla 15).

TABLA 15

Factores emocionales

\begin{tabular}{|c|c|}
\hline Informante & Frase \\
\hline Estudiante 15: & "Mentalizarme, ya que el traslado en el transporte público me da ansiedad por miedo al contagio" \\
\hline Estudiante 2: & $\begin{array}{c}\text { "Desconozco la ciudad, tendría que irme a alquilar, si no consigo un lugar cercano tendría } \\
\text { que viajar en autobús". }\end{array}$ \\
\hline
\end{tabular}

Fuente: Consulta a estudiantes y docentes. Elaboración propia (2021).

Algunos estudiantes señalan las dificultades de continuar con sus estudios si se retorna a la presencialidad, lo que desafía a la universidad a desarrollar estrategias administrativas como curriculares, con miras a evitar situaciones de exclusión CEPAL (2020) (tabla 16). 
TABLA 16

Dificultad para continuar con estudios

\begin{tabular}{ll}
\multicolumn{1}{c}{ Informante } & Frase \\
\hline Estudiante 7: & "No tengo opción" \\
Estudiante 92: & "Retiro de cursos" \\
\hline \multicolumn{2}{l}{ Fuente: Consulta a estudiantes y docentes. Elaboración propia (2021). }
\end{tabular}

Fuente: Consulta a estudiantes y docentes. Elaboración propia (2021).

El personal docente de la DET identificó como ventaja el ahorro de tiempo en traslados, en buscar aparcamientos y combustible, lo cual permite invertir más tiempo en labores académicas, repercutiendo en la mediación pedagógica y en la participación en múltiples actividades de formación. A su vez, se señala un mayor cumplimiento de los objetivos de los programas de estudio.

Por otro lado, la atención personalizada de estudiantes se vio favorecida por la inmediatez con que se atienden las solicitudes a través de los medios digitales. Asimismo, se enfatizó en la variedad y cantidad de estrategias didácticas aplicables en esta modalidad. Según León-León y Zúñiga-Meléndez (2019), la mediación pedagógica es un proceso potenciador de la creatividad a través de espacios de aprendizaje agradables donde se dé una comunicación en doble vía.

El aprendizaje de tecnologías para entornos educativos virtuales adquiere un carácter de obligatoriedad ante la situación de pandemia, ya que las personas deben buscar los medios necesarios para la autocapacitación en el manejo de herramientas innovadoras, favoreciendo su comprensión y la frecuencia de su uso.

Asimismo, se da un acercamiento entre las personas docentes y la población estudiantil en la atención de situaciones emocionales propias del estudiantado, favoreciendo la formación de vínculos y abriendo canales de comunicación. Por otro lado, se refieren a calidad de vida personal y familiar, dadas las mejores condiciones de autocuidado y de protección de sus familias.

En cuanto a las desventajas, según las personas docentes algunos cursos exigen la presencialidad según los contextos educativos. Otra de ellas, se asocia con la conectividad, principalmente por la calidad de los servicios, ya que la entrega de la docencia en entornos virtuales requiere de conexión de alta capacidad. Finalmente, las personas docentes, ante un eventual regreso a la presencialidad, necesitan recursos de conectividad y protocolos sanitarios en el campus.

Acerca de la preferencia por una u otra modalidad de trabajo, la población estudiantil se inclina por la modalidad presencial; mientras que las personas docentes se decantan por la presencialidad remota.

\section{SÍNTESIS Y REFLEXIONES FINALES}

Tanto personas estudiantes como docentes encuentran ventajas académicas la presencialidad remota; dichas ventajas se refieren a la socialización desde entornos virtuales, la mediación pedagógica, la disponibilidad de recursos didácticos virtuales, el desarrollo de habilidades profesionales (tecnológicas, comunicativas, creativas, autoconocimiento) y la posibilidad de matricular más cursos en un periodo (flexibilidad horaria).

Por otro lado, según algunas de las personas docentes se da un acercamiento con el estudiantado, principalmente por la frecuencia de los contactos virtuales sincrónicos, específicamente en horas de atención individual, ofrecida de manera permanente a lo largo de los cursos, hecho señalado como una ventaja. Sin embargo, se valora como desventaja, por parte de ambas poblaciones, la necesidad de interactuar 
físicamente, ya que los acercamientos personales permiten reconocer integralmente a la otra persona, por lo que al percibirla actúan todos los sentidos a la vez, en un acto de comunicación completo.

Ambos grupos se ven limitados al desarrollar las prácticas profesionales, especialmente aquellas en las cuales se requiere visitar a las poblaciones con las cuales se trabaja. Es una condicionante en términos del conocimiento de los procedimientos necesarios para ejecutar prácticas complementarias durante el aprendizaje conceptual y actitudinal.

La persona docente, desde la presencialidad remota, realiza tareas, cuya planificación resulta laboriosa en términos de tiempo y organización de estrategias y recursos, para la entrega de la docencia. Además de ello, las tareas asignadas a las personas estudiantes, según esta modalidad, se duplican para garantizar y dar cuenta de su proceso de aprendizaje.

Es importante reconocer la deuda histórica con algunos sectores sociales en materia de conectividad y acceso a la utilización de las herramientas tecnológicas aplicadas al proceso educativo. En tanto no se asuma el tema de conectividad como parte del derecho a la educación, esta brecha se tornará cada vez más grande entre los que tienen acceso al internet y a los recursos tecnológicos y quienes carecen de tales recursos. Lo anterior, involucra tanto a docentes como al estudiantado. En este sentido, también se reconocen los esfuerzos hechos por los diferentes departamentos de la UNA para responder con alternativas viables tanto para el profesorado como para el estudiantado, brindándole las condiciones idóneas para su desempeño durante el proceso educativo de entornos virtuales.

Es necesario reflexionar seriamente acerca los ajustes por realizar, en caso de retornar a las aulas, ya que tales ajustes presentan una mayor afectación del estudiantado, en aspectos como: el traslado, la inseguridad social, los horarios de trabajo, el gasto económico en transporte público y alimentación, la reestructuración en la distribución del tiempo, debido a los roles familiares y laborales, la dificultad en la búsqueda de cuidadores de niños o adultos mayores para remplazar a la persona estudiante durante sus horas de estudio.

La condición de género implica para las mujeres una mayor carga de trabajo, ellas desempeñan roles de madres o cuidadoras, dificultándoles realizar los ajustes necesarios para darle continuidad a sus estudios. Al respecto, deben realizarse esfuerzos que respondan a la política institucional para la equidad de género.

Con respecto a los ajustes, al momento de retornar a la presencialidad el estudiantado en condición laboral, que apoya económicamente a sus familias, se vería en la encrucijada de abandonar sus estudios o dejar de colaborar con la economía familiar. Por tanto, existe una posibilidad real de exclusión de estudiantes de las instituciones universitarias. Así las cosas, la modalidad es una de las condiciones que ameritan un ajuste significativo, tomando en consideración que fue gracias a la presencialidad remota que muchos estudiantes pudieron retomar los estudios, inscribirse en más cursos por ciclo lectivo o, incluso, acceder a empleos de jornadas completas o parciales.

Hay procesos personales de adaptación que resultan ante la migración de una modalidad a otra, algunas personas se inestabilizan ante los cambios, afectándolas en la toma de decisiones relacionadas con la organización del tiempo, el desplazamiento, el ajuste de jornadas laborales, la atención de asuntos familiares y la generación de espacios destinados a proteger la salud mental.

Las autoridades universitarias deben valorar y aplicar constantemente las medidas sanitarias y asegurar el apego a los protocolos, con miras conferir a las personas un sentido de seguridad en los espacios físicos, durante el desplazamiento y estancia dentro del campus universitario. A pesar de las experiencias estudiantiles positivas dadas a través de la presencialidad remota, para algunas personas estudiantes dicha modalidad no resultó amigable, y afrontaron dificultades durante su desempeño según esta modalidad. 
Las personas docentes refieren experiencias positivas en el trabajo presencial remoto, ya que optimizaron el tiempo, la preparación de las sesiones de trabajo, la diversificación de estrategias de mediación y los recursos digitales, así como la atención individual del estudiantado.

La presencialidad remota representa la modalidad de trabajo más segura y menos desfavorable para ambas poblaciones en términos personales y académicos, por lo cual sería ideal continuar apegándose a ella, en tanto se atiendan las condiciones óptimas de accesibilidad, favoreciendo así la continuidad de las actividades educativas y, por ende, garantizando el derecho a la educación.

El estudio concluye que la mediación pedagógica requiere de ajustes para responder a las demandas actuales, principalmente en cuanto a la incorporación de las TICs en los entornos de aprendizaje.

\section{REFERENCIAS}

Acosta, C. OrtegA, D. y Díaz, Y. (2020). Educación presencial con mediación virutal: Una experiencia de Honduras en tiempos de la COVID-19. RIDU. E-ISSN: 2223-2516. Vol. 14. №2, jul-dic. Perú. Recuperado de: https://doi.org/10.19083/ridu.2020.1229

AgUilaR, M. E. B. (2018). El estrés y su influencia en la calidad de vida. Multimed, 21(6). Recuperado de http://www.revmultimed.sld.cu/index.php/mtm/article/view/688/1054

BisqueRRA, R. (Coord.). (2014). Metodología de la investigación educativa. Madrid, España: Editorial La Muralla.

Castellano GiL, J. M. (2020). Equipamiento, uso y consumo de tic en estudiantes de educación en la universidad nacional de Costa Rica. Conrado, 16(72), 334-341. Recuperado de: http://scielo.sld.cu/ scielo.php?script=sci_arttext\&pid=S1990-86442020000100334

CEPAL-UNESCO. (2020, agosto). La educación en tiempos de la pandemia de COVID-19. https://repositorio.cepal.org/bitstream/handle/11362/45904/1/S2000510_es.pdf.

De Pablos Pons, J., Bravo, M. P. C., López-Gracia, A., \& Lázaro, I. G. (2019). Los usos de las plataformas digitales en la enseñanza universitaria. Perspectivas desde la investigación educativa. REDU: Revista de Docencia Universitaria, 17(1), 15. Recuperado de: https://dialnet.unirioja.es/servlet/ articulo?codigo $=6977320$

DUARTE, O. S. (2019). Contexto familiar y los factores intervinientes en el rendimiento académico del sujeto educativo: Aproximación diagnóstica. Polo del Conocimiento: Revista científico-profesional, 4(4), 25-38. Recuperado de https://dialnet.unirioja.es/servlet/articulo?codigo=7164410

Hernández, R. Fernández C. Baptista, P. (2014) Metodología de la Investigación. México, D.F. McGraw- Hill/ Interamericana Editores, S.A. de C.V.

IbÁÑEZ, F. (2020). Educación en línea, Virtual, a Distancia y Remota de Emergencia, ¿cuáles son sus características y diferencias?. Recuperado de https:/observatorio.tec.mx/edu-news/ diferencias-educacion-online-virtual-a-distancia-remota

INCIARTE-RodrIGUeZ, M. (2008). Competencias docentes ante la virtualidad de la educación superior. Télématique, 7(2),19-38. [fecha de Consulta 16 de Mayo de 2021]. ISSN: 1856-4194. Disponible en: https://www.redalyc.org/articulo.oa?id=78470202

KIKUT, L. (2020). Análisis de resultados de la evaluación de la virtualización de cursos de la UCR ante la pandemia por covid-19: Perspectiva Estudiantil. Centro de Evaluación Académica. Disponible en: http://repositorio.ucr.ac.cr/handle/10669/81216

LEÓN-León, G., \& ZúÑIGA-Meléndez, A. (2019). Mediación pedagógica y conocimientos científicos que utilizan una muestra de docentes de ciencias en noveno año de dos circuitos del sistema educativo 
costarricense, para el desarrollo de competencias científicas. Revista Electrónica Educare, 23(2), 1-24. https://doi.org/10.15359/ree.23-2.5

Ocaña-Fernández, Y., Valenzuela-Fernández, L., \& Morillo-Flores, J. (2020). La competencia digital en el docente universitario. Propósitos y Representaciones, 8(1). http://dx.doi.org/10.20511/pyr2020. v8n1.455.

Organización de las Naciones Unidas para la Educación, La CIencia y La Cultura. (2019, mayo). INFRAESTRUCTURA $Y$ CONECTIVIDAD. IIPE-UNESCO. https://siteal.iiep.unesco.org/sites/default/files/sit_informe_ pdfs/siteal_infraestructura_y_conectividad_20190607.pdf

Ortiz, A. M. R., \& NeirA, E. A. S. (2018). Interactividad e interacción social: procesos esenciales en educación a distancia. Revista Virtual Universidad Católica del Norte, (55), 110-127.a distancia. Revista Virtual Universidad Católica del Norte, 55, 110-127.Recuperado de: https://revistavirtual.ucn.edu. co/index.php/RevistaUCN/article/view/999

Pablo Becher, P. B. (2020). Educação em tempos de pandemia. Condições de trabalho e percepções sobre o trabalho virtual de professores na cidade de bahia blanca (Argentina).Revista Educ@ção Científica, 4(8), 922-945. https://doi.org/10.46616/rce.v4i8.116

Rodriguez, A. (2018). Interactividad e interacción social: procesos esenciales en educación a distancia. Revista virtual Universidad Católica del Norte. Núm. 55 pág 110-127. Recuperado de: https://revistavirtual.ucn.edu.co/index.php/RevistaUCN/article/view/999

Rodriguez, V. (2021). Docencia Universitaria en medio del Covid-19. Adaptación y desarrollo de un curso de Bibliotecología en la virtualidad. Universidad de Buenos Aires. Disponible en: https://doi.org/10.34096/ics.i44.9818.

SHIMSHON, G. y Dye, D. (2020). El aprendizaje remoto es más que tecnología educativa. Recuperado de: https://observatorio.tec.mx/edu-news/aprendizaje-remoto-mas-que-tecnologa-educativa-opinion

SMITH, M. (2019). Las emociones de los estudiantes y su impacto en el aprendizaje: Aulas emocionalmente positivas (Vol. 157). Narcea Ediciones. Recuperado de https://scholar.google.es/scholar?as_ylo $=2017 \& q=$ factores+emocionales+que+afectan+el+aprendizaje\&hl=es\&as_sdt=0,5

TiJo, S. (2020). Enseñanza remota de emergencia en ingeniería civil: lecciones aprendidas. Encuentro Internacional de Educación en Ingeniería. Recuperado de https://acofipapers.org/index.php/ eiei/article/view/781

Universidad Nacional de Costa Rica. (2020, 10 de junio). Consejo Académico. Acuerdo №2. Art. III, inciso 1. Acta n¹9-2020. Comunicado en UNA-CONSACA-ACUE-118-2020.

Universidad Nacional de Costa Rica. (2020, 20 de marzo). Consejo Académico. Acuerdo N²2. Comunicado en UNA-CONSACA-ACUE-045-2020. 